\title{
Aneurisma de la aorta abdominal infectado por Campylobacter fetus. Comunicación de un caso clínico y revisión de la literatura médica
}

\author{
Abdominal aortic aneurysm infected with Campylobacter fetus. \\ Case report and medical literature review
}

Martín Milanesio', Rodrigo Alonso ${ }^{1}$, María Teresita Cornavaca', Flavio Gabriel Lipari² y Matías Gabriel Zanuzzi

'Servicio de Clínica Médica. Hospital Privado Universitario de Córdoba. Córdoba Capital, Argentina.

${ }^{2}$ Servicio de Infectología. Hospital Privado Universitario de Córdoba. Córdoba Capital, Argentina.

Recibido: 8 de septiembre de 2020 / Aceptado: 15 de abril de 2021

\section{Resumen}

La infección es una complicación infrecuente de los aneurismas de la aorta abdominal. Campylobacter fetus tiene un especial tropismo por el endotelio vascular y ha sido reportado como causa de infección de aneurismas aórticos. Este tipo de infección es de alta mortalidad por lo que el reconocimiento temprano con el inicio precoz de terapia antibacteriana efectiva es clave. Además del tratamiento médico, puede requerirse cirugía, la que tiene una alta letalidad en pacientes inestables y con comorbilidades. Comunicamos el caso clínico de un adulto mayor con un aneurisma de aorta abdominal infectado por $C$. fetus. Dado su compromiso del estado general y antecedentes cardiovasculares se decidió tratamiento médico con imipenem, con una buena respuesta clínica y microbiológica, sin recurrencia de los síntomas. También se presenta una revisión de los casos publicados.

Palabras claves: aneurisma infectado; Campylobacter fetus; aorta abdominal; aortitis.

\section{Introducción}

$\mathrm{L}$

os aneurismas de la aorta abdominal son dilataciones focales del segmento inferior de la aorta. Su infección es una complicación poco frecuente ${ }^{1}$ que se describe principalmente en personas de la tercera edad con algún grado de inmunosupresión ${ }^{2-4}$. La sobreinfección puede ser causada por varios mecanismos: la inoculación directa del microorganismo, siembra bacterémica, infección contigua o émbolos sépticos secundarios a una endocarditis ${ }^{5}$. Los microorganismos más frecuentemente descritos son Staphylococcus aureus y Salmonella spp ${ }^{6}$, siendo Campylobacter fetus un agente infrecuente 5 .

\begin{abstract}
Infection is a rare complication of abdominal aortic aneurysms. Campylobacter fetus has special tropism for vascular endothelium. It has been reported as a cause of infected abdominal aortic aneurysms. The mortality of these patients is high, so an early recognition with a start of antibiotic therapy is crucial. In addition to medical treatment surgery may be required, which has high mortality in patients with many diseases and unstable. We report the case of an old man with infected abdominal aortic aneurysm with C. fetus, whom was decided to be treated with imipenem, due to his cardiovascular history and his general condition at admission. The patient showed a good clinical response without recurrence of symptoms. We also carry out a review of the reported cases.

Key words: infected aneurysm; Campylobacter fetus; abdominal aorta; aortitis.
\end{abstract}

Clínicamente se manifiesta por la tríada característica de fiebre, dolor abdominal o lumbar y masa abdominal pulsátil. El hallazgo más frecuente en los exámenes de laboratorio es la leucocitosis?

El diagnóstico se confirma con el aislamiento del microorganismo en hemocultivos y/o en la pieza quirúrgica ${ }^{7}$ con imágenes compatibles en la TC abdominal ${ }^{8-10}$.

Dada su alta mortalidad ${ }^{11}$ se debe iniciar terapia antibacteriana intravenosa inmediata. Si bien se recomienda la cirugía, la mortalidad posterior a ésta puede ser hasta $31 \%$, con pobres resultados ${ }^{12}$, por lo que en pacientes inestables, con múltiples comorbilidades y que respondan bien a la terapia médica inicial, el tratamiento quirúrgico podría ser diferido 5 . 
Presentamos el caso de un paciente con un aneurisma de la aorta abdominal infectado por C. fetus, el cual se presentó con un cuadro de sepsis, con buena respuesta al tratamiento médico.

\section{Caso clínico}

Varón de 76 años de edad, con antecedentes de un aneurisma aórtico infrarrenal, tabaquismo, hipertensión arterial, cardiopatía isquémica con tres bypass aortocoronarios, enfermedad pulmonar obstructiva crónica y enfermedad celíaca no adherente a la dieta. Acudió al servicio de urgencia por fiebre de una semana de evolución, acompañado de molestias abdominales inespecíficas en la región del mesogastrio, astenia y anorexia que se habían acentuado en los últimos dos días. Refirió un episodio de diarrea autolimitada no disentérica siete días previo al inicio del cuadro actual, la que asoció a su enfermedad celíaca. No refirió sintomatología respiratoria, urinaria o cardíaca.

Al examen físico se encontraba en mal estado general, con temperatura axilar de $38,5^{\circ} \mathrm{C}$, presión arterial de $90 / 40$ $\mathrm{mmHg}$, frecuencia cardíaca de 105 latidos por minuto y saturación de oxígeno ambiental de 92\%. Estaba lúcido, orientado, con mucosas secas y signos de hipoperfusión periférica. Su abdomen era blando y se palpaba una masa pulsátil dolorosa en la región mesogástrica. El resto del examen no presentaba anormalidades.

En el laboratorio de ingreso destacaba un hemograma con leucocitosis (23.500 céls $\left./ \mathrm{mm}^{3}\right)$, PCR 5,8 mg/dl (normal hasta $0,5 \mathrm{mg} / \mathrm{dl}$ ), VHS $60 \mathrm{~mm} / \mathrm{h}$, ácido láctico elevado y función renal, enzimas pancreáticas y hepáticas normales. El examen de orina y radiografía de tórax eran normales y las serologías virales (VIH, VHB y VHC) fueron no reactivas.

Se realizó un TC de abdomen con contraste intravenoso donde se observó un aneurisma aórtico infrarrenal trombosado de $7 \mathrm{~cm}$ de ancho, con la pared de aspecto inflamatorio, tumefacción de la grasa circundante y adenopatías locales reactivas, compatibles con un aneurisma infectado (Figura 1).

Ante la sospecha de una sepsis con un probable foco aórtico se inició terapia empírica con piperacilinatazobactam 4,5 g cada $8 \mathrm{~h}$, por $72 \mathrm{~h}$.

Se realizaron pruebas para descartar una causa inmunológica (anticuerpos antinucleares, anticuerpos anticitoplasma de neutrófilos, factor reumatoídeo y complemento) junto con una ecografía doppler de arterias temporales, los cuales fueron normales.

Los dos hemocultivos tomados previo al inicio de la terapia antibacteriana fueron informados a las $72 \mathrm{~h}$ como positivos a bacilos gramnegativos e identificados por la técnica MALDI-TOF MS como Campylobacter fetus. La susceptibilidad a los antimicrobianos se determinó

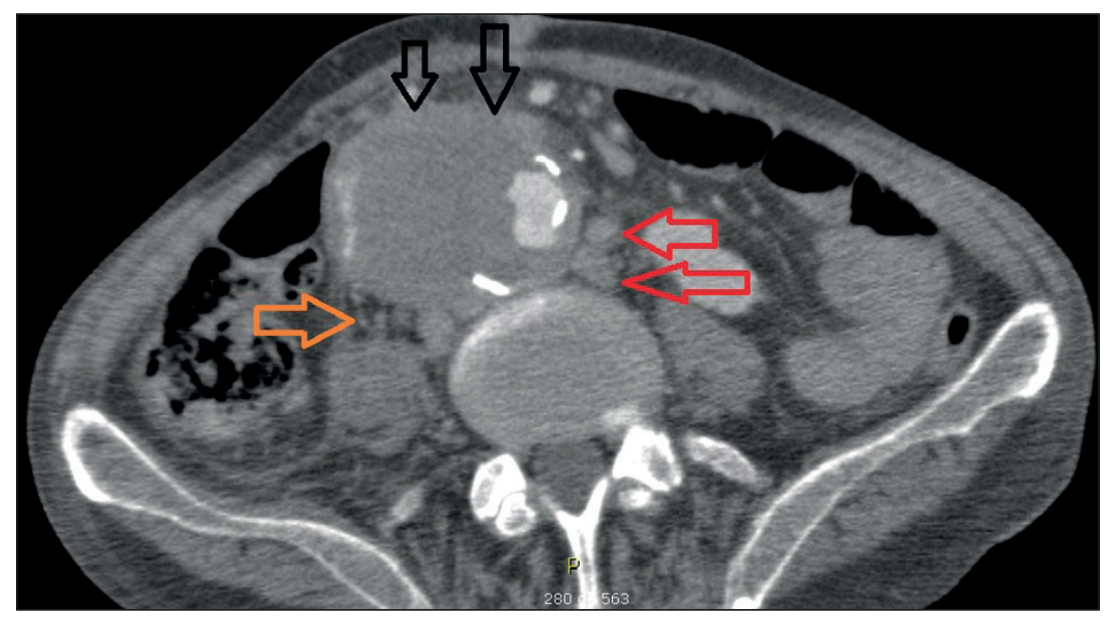

Figura 1. TC de abdomen corte axial donde se observa un aneurisma de la aorta abdominal infrarrenal trombosado con engrosamiento de su pared que capta contraste (flechas negras), tumefacción de la grasa circundante (flecha naranja) y múltiples adenopatías reactivas periaórticas (flechas rojas).

por el método de difusión con discos en agar Müeller Hinton, suplementado con $5 \%$ de sangre de cordero. Al no contar con puntos de corte CLSI para aislados de $C$. fetus extraintestinales, utilizamos los de enterobacterias CLSI M100, según lo recomendado por protocolo Red Whonet, Argentina. La cepa fue sensible a imipenem, ciprofloxacina, amoxicilina-clavulánico, gentamicina y cefepime. Se ajustó antibioticoterapia a imipenem intravenoso $1 \mathrm{~g}$ cada $8 \mathrm{~h}$, durante siete días.

Tras el aislamiento microbiológico se indagaron sobre posibles fuentes de contagio, como exposición a aves o consumo de alimentos de riesgo, las cuales fueron negadas por el paciente.

El ecocardiograma transtorácico descartó la presencia de vegetaciones. Los hemocultivos de control al quinto día fueron negativos. El paciente mejoró sus síntomas y los parámetros inflamatorios descendieron, por lo que tras siete días de imipenem se decidió el alta continuando la terapia con ciprofloxacina $500 \mathrm{mg}$ cada $12 \mathrm{~h}$, por vía oral, hasta completar veintiocho días de tratamiento. El paciente no tenía contraindicación ni medicación que presentara interacción con las quinolonas.

En controles posteriores el paciente no tuvo recurrencia de los síntomas, por lo que tras resuelto el proceso infeccioso fue derivado al servicio de hemodinamia para colocación de una endoprótesis vascular a la cual se negó. No se realizó TC de control ante la negativa del paciente.

\section{Discusión}

La infección de los aneurismas de la aorta abdominal tiene una incidencia de 0,5 a $2 \%$ y una mortalidad aproximada de $30 \%{ }^{11}$, siendo Campylobacter fetus una etiología poco frecuente. 
Campylobacter fetus es un bacilo gramnegativo microaerófilo con especial tropismo por el endotelio vascular ${ }^{13}$. Se puede identificar por su capacidad de crecer a $25^{\circ} \mathrm{C}$ y $37^{\circ} \mathrm{C}$ y no a $42^{\circ} \mathrm{C}^{3,13}$. Del punto de vista bioquímico se caracteriza por ser oxidasa y catalasa positiva ${ }^{14}$. Su forma de transmisión está asociada a la ingesta de agua o alimentos contaminados de origen animal y afecta principalmente a personas de sexo masculino, de la tercera edad y con múltiples comorbilidades ${ }^{3,4,15}$.

Nuestro paciente era añoso, con varias patologías asociadas y con el antecedente de un aneurisma aórtico abdominal, todos factores que favorecieron la infección por C. fetus. No encontramos una posible fuente de contagio. Clínicamente se presentó con la tríada clásica, la que se encuentra presente en menos de $30 \%$ de los casos ${ }^{9}$, acompañado de un aumento de los parámetros inflamatorios?

Los hallazgos imagenológicos son claves en el diagnóstico y seguimiento de esta patología. Dentro de estos, destacan el engrosamiento de la pared arterial y edema periaórtico, con forma de aneurisma sacular o lobulado, gas ectópico que rodea a la aorta ${ }^{8} \mathrm{y}$ aumento de la grasa periaórtica ${ }^{9}, 10$. En caso de disponibilidad de medicina nuclear, la tomografía por emisión de positrones/tomografía computada con fluorodesoxiglucosa F-18 (PET/CT con 18-FDG) presenta una alta sensibilidad y especificidad para el diagnóstico de procesos inflamatorios aórticos, ya sea infecciosos o de otra etiología. Permite demostrar la mayor captación del radiofármaco en la pared aórtica mientras la actividad inflamatoria permanezca activa y una disminución progresiva de ésta con la terapia antibacteriana efectiva, lo que es útil en el seguimiento ${ }^{16}$.

El principal diagnóstico diferencial es la vasculitis de grandes vasos, la que también puede presentarse con fiebre y aumento de los parámetros inflamatorios ${ }^{17}$, aunque suele tener otras manifestaciones sistémicas como cefalea hemicránea, alteraciones visuales, claudicación mandibular, alteración en el pulso radial y los cultivos son negativos ${ }^{17}$. Nuestro paciente no presentaba síntomas sistémicos compatibles con vasculitis, y se realizaron métodos complementarios (doppler de arteria temporal y panel reumatológico) que descartaron una patología autoinmune.

En nuestro caso, dada la alta sospecha de un aneurisma sobreinfectado por la clínica e imágenes, se inició tratamiento antibacteriano precoz que permitió un rápido control del proceso infeccioso. Posteriormente, con la identificación microbiológica se pudo ajustar el tratamiento y realizar un adecuado paso a terapia oral.

Dada la escasa cantidad de casos publicados, ni ensayos clínicos que comparen la eficacia de diferentes antibacterianos, no existe un consenso para el tratamiento de este tipo de infección. En una revisión de la susceptibilidad antimicrobiana de 111 aislados de C. fetus no hubo resistencia a ampicilina, gentamicina e imipenem, siendo éste último el que presentó la menor concentración inhibitoria mínima de $90 \%(\mathrm{CIM} 90)^{18}$. En dicho estudio, la resistencia a ciprofloxacina y tetraciclina fue de 3 y $34 \%$, respectivamente ${ }^{18}$. En otra serie clínica de aortitis por $C$. fetus ${ }^{7}$, imipenem fue el antibacteriano que logró la mayor tasa de curaciones ( $100 \%$ de los pacientes).

La terapia debe iniciarse con antibacterianos por vía intravenosa hasta que los parámetros inflamatorios desciendan, tras lo cual se puede pasar a la vía oral ${ }^{7}$. Dentro de estas opciones se encuentran amoxicilina/ácido clavulánico y ciprofloxacina. Aunque se demuestre sensibilidad a eritromicina y cefalosporinas, no se recomienda su uso para infecciones graves ya que se han asociado a un mayor fracaso terapéutico ${ }^{18}$.

Si bien no encontramos guías que recomienden la realización de ecocardiografía de rutina en pacientes con bacteriemia por C. fetus, dado el tropismo de este microorganismo por el endotelio vascular, debe descartarse la endocarditis infecciosa como posible fuente de bacteriemia. Se han reportado casos clínicos de endocarditis secundarias a $C$. fetus, requiriendo la mayoría tratamiento quirúrgico ${ }^{19-21}$. En nuestro paciente se descartó la endocarditis con una ecocardiografía transtorácica. Probablemente, el episodio previo de diarrea pudo ser el origen de la bacteriemia que permitió la infección de la pared aneurismática.

Dada la alta mortalidad asociada, se recomienda la cirugía de urgencia en casos de refractariedad al tratamiento médico. La técnica quirúrgica convencional en estos casos es la escisión de todos los tejidos infectados y la ligadura de la aorta infrarrenal, seguida de una reconstrucción arterial extra-anatómica? ${ }^{7}$. Otros procedimientos quirúrgicos incluyen la reconstrucción in situ con aloinjertos criopreservados o con venas profundas de las extremidades inferiores? ${ }^{7}$. En pacientes con alto riesgo quirúrgico, la reparación endovascular mediante hemodinamia es una alternativa, pero en el contexto de una bacteriemia se asocia a un mayor riesgo de infección del material protésico ${ }^{22}$.

En nuestro caso, dado que el paciente presentaba múltiples comorbilidades con alto riesgo quirúrgico y una buena respuesta a la antibioticoterapia inicial, se decidió diferir la cirugía. Tras la resolución del proceso infeccioso y dado los antecedentes del paciente se optó por el tratamiento endovascular, sin embargo, el paciente se negó.

Realizando una búsqueda bibliográfica en la base de datos científica PubMed, en idioma español e inglés, encontramos 32 casos reportados de aneurismas de la aorta abdominal infectados por C. fetus, desde los años 1971 a 2016, los que se resumen en la Tabla $1^{5,7,9,11,13,15,23-38}$. El 90\% de los pacientes eran varones, la mayoría con comorbilidades. La edad promedio fue de 69 años, con un rango de 45 a 91 . Sólo seis pacientes eran menores de 60 años. Los síntomas más frecuentes fueron fiebre y 


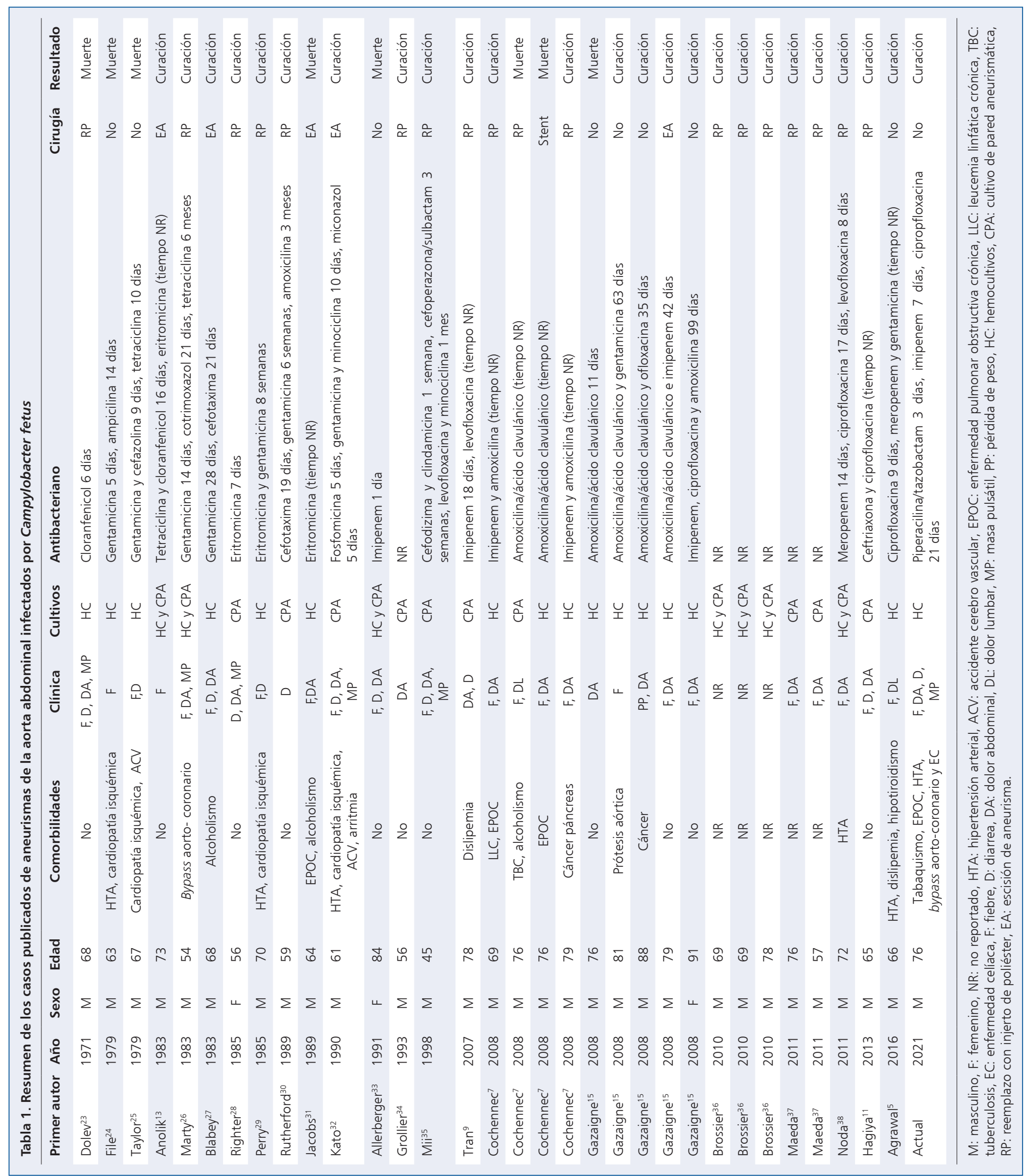




\section{- Crci caso clinico}

dolor abdominal, presentes en 72 y $66 \%$ de los pacientes; respectivamente. Sólo en cinco pacientes se palpaba una masa pulsátil, siendo los únicos que presentaron la tríada clínica completa. Un tercio tenía antecedentes de diarrea. El 69\% tenía hemocultivos positivos y el microorganismo se aisló de la pared del aneurisma en sólo 17 pacientes. Todos los pacientes recibieron antibacterianos. Tanto la elección como la duración de los mismos fueron variados, siendo los más usados amoxicilina, gentamicina, imipenem y ciprofloxacina. Ocho pacientes no se sometieron a cirugía, cuatro de ellos por fallecimento antes del procedimiento. La técnica quirúrgica más utilizada fue el reemplazo con injerto de poliéster (56\%). En total, ocho pacientes fallecieron.

\section{Referencias bibliográficas}

1.- Bouzas M, Ponte C, Van Houte B, Vasquez C. Mycotic aortic aneurysms treated by endovascular repair: initial experience in a single center. Acta Chir Belg 2019;119: 189-94. doi: 10.1080/00015458.2018.1432741.

2.- Oderich G S, Panneton J M, Bower T C, Cherry K J, Rowland C M, Noel A A, et al. Infected aortic aneurysms: aggressive presentation, complicated early outcome, but durable results. J Vasc Surg 2001; 34: 900-8. doi: 10.1067/ mva.2001.118084.

3.- Fica A, Illanes V, Sakurada A, Vidal M, Valenzuela ME. Bacteriemia por Campylobacter fetus subsp fetus en un paciente inmunosuprimido. Rev Chilena Infectol 2006; 23: 336-9. doi: 10.4067/S071610182006000400007.

4.- Fica A, Porte L, Braun S, Veas N, Pavez C, Dabanch J, et al. Bacteriemias e infección endovascular por Campylobacter spp: nuestra experiencia en un cuarto de siglo de historia. Rev Chilena Infectol 2011; 28: 211-6. doi: 10.4067/S0716-10182011000300002.

5.- Agrawal A, Sikachi R R. Infective abdominal aortitis due to Campylobacter fetus bacteremia: A case report and review of literature. Intractable Rare Dis Res 2016; 5: 290-3. doi: 10.5582/irdr.2016.01059.

6.- Brown S L, Busuttil R W, Baker J D, Machleder H I, Moore W S, Barker W F. Bacteriologic and surgical determinants of survival in patients with mycotic aneurysms. J Vasc Surg 1984;1: 541-7. doi: 10.1016/07415214(84)90040-5.

7.- Cochennec F, Gazaigne L, Lesprit P, Desgranges P, Allaire E, Becquemin J P. Aortoiliac aneurysms infected by Campylobacter fetus. J Vasc Surg 2008; 48: 815-20. doi: 10.1016/j.jvs.2008.05.076.

8.- Macedo T A, Stanson A W, Oderich G S, Johnson C M, Panneton J M, Tie M L. Infected aortic aneurysms: imaging findings. Radiology 2004; 231: 250-7. doi: 10.1148/ radiol.2311021700.

9.- Tran J K, de Virgilio C. Management of an abdominal aortic aneurysm infected with Campylobacter fetus: a case report. Ann Vasc Surg 2007; 21: 137-42. doi: 10.1016/j. avsg.2007.01.002.
10.- Peralta Moscoso M T, García López M, Vilariño Rico J. Mycotic aneurysm of the abdominal aorta caused by Campylobacter fetus. Cir Esp 2015; 93: 413-5. doi: 10.1016/j. cireng.2015.05.009.

11.- Hagiya H, Matsumoto M, Furukawa $H$, Murase T, Otsuka F. Mycotic abdominal aortic aneurysm caused by Campylobacter fetus: a case report and literature review. Ann Vasc Surg 2014; 28: 1933.e7-1933.e14. doi: 10.1016/j. avsg.2014.06.072.

12.- Reddy D J, Shepard A D, Evans J R, Wright D J, Smith R F, Ernst C B. Management of infected aortoiliac aneurysms. Arch Surg 1991;126: 873-9. doi: 10.1001/ archsurg.1991.01410310083012.

13.- Anolik J R, Mildvan D, Winter J W, Puttlitz D, Rubenstein S, Lozman H. Mycotic aortic aneurysm: A complication of Campylobacter fetus septicemia. Arch Intern Med 1983;143: 609-10. doi: 10.1001/ archinte.1983.00350030223047.

14.- Lawrence G D, Biggs Jr. R D, Woodward T E. Infection caused by Vibrio fetus. Report of two cases. Arch Intern Med 1967;120: 459-64. doi: 10.1001/archinte.1967.04410010073010.

15.- Gazaigne L, Legrand P, Renaud B, Bourra B, Taillandier E, Brun-Buisson C et al Campylobacter fetus bloodstream infection: risk factors and clinical features. Eur J Clin Microbiol Infect Dis 2008; 27:185-9. doi: 10.1007/s10096-007-0415-0.

16.- Bruls S, Courtois A, Nusgens B, Defraigne J O, Delvenne P, Hustinx R, et al.18F-FDG $\mathrm{PET} / \mathrm{CT}$ in the management of aortitis. Clin Nucl Med 2016; 41: 28-33. doi: 10.1097/ RLU.0000000000001016.

17.- Sharma S, Pandey N N, Sinha M, Chandrashekhara S H. Etiology, diagnosis and management of aortitis. Cardiovasc Intervent Radiol 2020; 43: 1821-36. doi: 10.1007/ s00270-020-02486-6.

18.- Tremblay C, Gaudreau C, Lorange M. Epidemiology and antimicrobial susceptibilities of 111 Campylobacter fetus subsp. fetus strains isolated in Québec, Canada, from 1983 to 2000. J Clin Microbiol. 2003; 41: 463-6. doi: $10.1128 / \mathrm{jcm} .41 .1 .463-466.2003$.

19.- Petridou C, Strakova L, Simpson R. Campylobacter fetus prosthetic valve endocarditis presenting as a stroke. JMM
Case Rep 2018; 5: e005147. doi: 10.1099/ jmmcr.0.005147.

20.- Farrugia D C, Eykyn S J, Smyth E G. Campylobacter fetus endocarditis: two case reports and review. Clin Infect Dis 1994; 18 : 443-6. doi: 10.1093/clinids/18.3.443.

21.- Haruyama A, Toyoda S, Kikuchi M, Arikawa T, Inami S, Otani N, et al. Campylobacter fetus as cause of prosthetic valve endocarditis. Tex Heart Inst J. 2011; 38: 584-7.

22.- Dimitrief M, Cherbanyk F, Déglise S, Pezzetta E. Contained rupture of a mycotic infrarenal aortic aneurysm infected with Campylobacter fetus. BMJ Case Rep 2016; 2016:bcr2016215582. doi: 10.1136/bcr-2016215582.

23.- Dolev E, Altmann G, Padeh B. Vibrio fetus septicemia. A case report. Isr J Med Sci 1971; 7: 1188-91. doi: 10.1016/0741-5214(89)903558.

24.- File T M Jr, Barnishan J, Fass R J. Campylobacter fetus sepsis with mycotic aortic aneurysm. Arch Pathol Lab Med 1979; 103: 143-5.

25.- Taylor P R, Weinstein W M, Bryner J H Campylobacter fetus infection in human subjects: association with raw milk. Am J Med 1979; 66: 779-83. doi: 10.1016/00029343(79)91116-1.

26.- Marty A T, Webb T A, Stubbs K G, Penkava R R. Inflammatory abdominal aortic aneurysm infected by Campylobacter fetus. JAMA 1983; 249: $1190-2$.

27.- Blabey R G Jr, Parry M F, Bull S M, Weed C B. Mycotic aneurysm of the abdominal aorta: successful management of Campylobacter fetus aortitis. Conn Med 1983; 47: 129-30.

28.- Righter J, Woods J M. Campylobacter and endovascular lesions. Can J Surg 1985; 28 : 451-2.

29.- Perry M O. Infected aortic aneurysms. J Vasc Surg 1985; 2: 597-9. doi: 10.1067/mva.1985. avs0020597.

30.- Rutherford E J, Eakins J W, Maxwell J G, Tackett A D. Abdominal aortic aneurysm infected with Campylobacter fetus subspecies fetus. J Vasc Surg 1989; 10: 193-7.

31.- Jacobs J, Van Lierde J, Nevelsteen A, Vermylen J, Verbist L. Campylobacter fetus subspecies fetus infection of an abdominal aneurysm. Acta Clin Belg 1989; 44: 123-8. 


\section{Caso Clínico}

doi: 10.1080/17843286.1989.11717999.

32.- Kato R, Ohta T, Kazui H, Kondo M, Naiki K, Tsuchioka H. Campylobacter fetus infection of abdominal aortic aneurysm. J Cardiovasc Surg (Torino) 1990; 31: 756-9.

33.- Allerberger F, Kasten M J, Anhalt J P. Campylobacter fetus subspecies fetus infection. Klin Wochenschr 1991; 69: 813-6. doi: 10.1007/BF01744276.

34.- Grollier G, Burucoa C, Ricco J B, Pezennec L, Fauchère $\mathrm{J} \mathrm{L}$. Isolation and immunogenicity of Campylobacter fetus subsp. fetus from an abdominal aortic aneurysm. Eur J Clin
Microbiol Infect Dis 1993; 12: 847-9. doi: 10.1007/BF02000405.

35.- Mii S, Tanaka K, Furugaki K, Sakata H, Katoh $\mathrm{H}$, Mori A. Infected abdominal aortic aneurysm caused by Campylobacter fetus subspecies fetus: report of a case. Surg Today 1998; 28 : 661-4. doi: 10.1007/s005950050204.

36.- Brossier J, Lesprit P, Marzelle J, Allaire E, Becquemin J P, Desgranges P. New bacteriological patterns in primary infected aorto-iliac aneurysms: a single-centre experience. Eur J Vasc Endovasc Surg 2010; 40: 582-8. doi: 10.1016/j.ejvs.2010.07.020.
37.- Maeda H, Umezawa H, Goshima M, Hattori T, Nakamura T, Umeda T, et al. Primary infected abdominal aortic aneurysm: surgical procedures, early mortality rates, and a survey of the prevalence of infectious organisms over a 30-year period. Surg Today 2011; 41: 346-51. doi: 10.1007/s00595-010-4279-z.

38.- Noda Y, Sawada K, Yoshida S H, Nishida S, Yamamoto S, Otake Y, et al. Mycotic abdominal aneurysm caused by Campylobacter fetus: a case report for surgical management. Ann Vasc Dis 2011; 4: 56-9. doi: 10.3400/avd. cr.10.01028. 